\title{
Modelo matemático para determinar presión permitida de pérdidas de filtrado de lodo controladas
}

\author{
Diego Armando Vargas Silva ${ }^{1 *}$; Zuly Calderón Carrillo후 Darwin Mateus Tarazona² \\ ${ }^{1}$ Escuela de Ingeniería de Petróleos. Universidad Industrial de Santander (UIS). Carrera 27 Calle 9. Bucaramanga, Colombia. \\ ${ }^{2}$ Instituto Colombiano del Petróleo (ICP). Vía Piedecuesta Km 7. Piedecuesta, Colombia. \\ *E-mail:diegoavargass@hotmail.com
}

\begin{abstract}
Resumen
Durante una perforación es de gran importancia tener una ventana de estabilidad lo más precisa posible para evitar problemas como colapso del pozo, influjos, pérdidas de circulación o de filtrado, entre otros. Aunque la geomecánica permite determinar una densidad óptima, en la realidad pueden existir formaciones permeables por las cuales pueden ocurrir pérdidas de filtrado considerables lo cual aumenta los costos. En literatura existe un modelo matemático que proviene de la ley de continuidad, una ecuación de estado y una ecuación de flujo (Darcy) el cual es utilizado en análisis de pruebas de presión, analizando la presión necesaria para tener flujo de la formación hacia el pozo. El objetivo de esta investigación es demostrar que este modelo se puede implementar para estimar la presión permitida del lodo durante la perforación y controlar el filtrado del este fluido hacia la formación para de esta forma, adicionar una nueva curva a la ventana de estabilidad. Para lograr tal objetivo se utilizaron modelos y correlaciones y se dio un estimado de las propiedades de la formación en función de la profundidad y con un caudal máximo permitido de pérdidas de filtrado, se determinó la presión adicional a la presión de formación con la cual se puede perforar sin presentar grandes pérdidas de filtrado disminuyendo los costos. Finalmente, se hizo un análisis de sensibilidad concluyendo que la permeabilidad y la viscosidad, son las variables más importantes del modelo.
\end{abstract}

Palabras claves: Geomecánica, Pérdida de circulación, Filtrado.

\section{Mathematical model to determine permissible pressure loss of mud controlled filtering}

\begin{abstract}
During a drilling, it is very important to have a stability window as accurate as possible to avoid problems such as collapse of the well, loss of circulation or filtration, among others. Although geomechanics makes it possible to determine an optimum density, in reality there may be permeable formations whereby considerable filtration losses can occur which increases costs and NPT. In literature there is a mathematical model that comes from the law of continuity, a state equation and a flow equation (Darcy) which is used in pressure test analysis, analyzing the pressure needed to have flow from the formation to the well . The objective of this research is to demonstrate that this model can be implemented to estimate the allowed pressure of the sludge during the drilling and to control the filtration of this fluid towards the formation in order to add a new curve to the stability window. To achieve this objective, models and correlations were used and an estimate of the properties of the formation as a function of depth and with a maximum allowed filtration loss rate was determined, the additional pressure was determined with which It can perforate without presenting great loss of filtration reducing costs. Finally, a sensitivity analysis was made concluding that permeability and viscosity are the most important variables of the model.
\end{abstract}

Keywords: Geomechanics, Circulation lost, Filtered.

\section{Introducción}

En un proyecto de perforación, la densidad de lodo utilizada debe mantener una presión superior a la presión de poro para evitar influjos y a la vez evitar fracturar la formación (Campana, \& Tapia, 2017). A pesar de estar dentro de estos rangos seguros, existen zonas fracturadas y permeables a través de las cuales pueden ocurrir pérdidas de circulación y filtrado como se enuncia en el trabajo de Ghalambor, Salehi, Shahri

Cita: Vargas, D., Calderón, Z. y Mateus, D. (2018). Modelo matemático para determinar presión permitida de pérdidas de filtrado de lodo controladas. Revista Fuentes: El reventón energético, 16(1), 47-55. 
\& Karimi (2014); entre los trabajos que analizan las pérdidas de circulación se encuentran los propuestos por Sanfillippo, Brignoli, Santarelli \& Bezzola,(1997) y Shahri, Zeyghami \& Majidi (2012); esta investigación se centró en analizar las pérdidas por filtrado, estas pérdidas, especialmente en arenas pueden convertirse en un problema grave ya que el aumento en el espesor de la torta puede generar diversos problemas tales como: pega diferencial, puntos apretados, dificultad en la cementación, disminución del espacio anular $\mathrm{y}$ aumento en los costos de perforación (Manual Fluidos de Perforación MI SWACO 2001). Para evitar este problema se pretende implementar un modelo matemático para determinar la presión permitida para mantener controladas las pérdidas de filtrado de lodo e incluir una nueva curva de esta presión al modelo de estabilidad (Portilla, Suárez \& Corzo, 2012). Para derivar esta curva hay que partir de la ley de Darcy en flujo radial en incluir el efecto de reducción de permeabilidad debido al daño en la cara del pozo, cabe resaltar que este daño se origina por el incremento del espesor de la torta (Ayala, Torres, Valencia \& Loaiza, 2016). Para cumplir con el objetivo planteado se revisaron modelos matemáticos propuestos en la literatura dentro de los que se resalta el propuesto por Parn-Anurak \& Engler (2005) el cual basa su teoría en un balance de masa de la torta en cuanto a la tasa de crecimiento y erosión de esta. Al final llega a un modelo iterativo por lo cual no fue implementado. Por otra parte, un modelo analítico utilizado en análisis de pruebas de presión que parte de un balance de masa (Escobar, 2003), cumple con condiciones y características necesarias para analizar cambios de presión en la cara del pozo. Por lo tanto, como aporte de esta investigación, este modelo será implementado para analizar flujo de filtrado de lodo del pozo hacia la formación.

Para solucionar este modelo se deben estimar las propiedades de la formación en función de la profundidad tales como porosidad, permeabilidad, viscosidad, compresibilidad, temperatura y parámetros como caudal de filtrado, tiempo de exposición y radio de pozo, posteriormente se estimará la presión máxima permitida con pérdidas de filtrado de lodo controlada la cual permitirá de esta forma complementar la ventana de estabilidad con la nueva curva.

\section{Fundamentos teóricos de filtrado de lodo}

Los fluidos de perforación se componen de una fase líquida y partículas sólidas. La filtración se refiere a la acción mediante la cual la presión diferencial hace entrar a la fase líquida del lodo de perforación dentro de una formación permeable (Ayala, Gómez \& León, 2011); durante este proceso, las partículas sólidas son filtradas, formando un revoque como se observa en la Figura 1. La filtración ocurre bajo condiciones tanto dinámicas como estáticas, durante las operaciones de perforación. La filtración bajo condiciones dinámicas ocurre mientras el fluido de perforación está circulando, mientras que la filtración estática ocurre en otros momentos como por ejemplo: durante las conexiones, los viajes o cuando el fluido no está circulando. (Manual Fluidos de Perforación MI SWACO 2001).

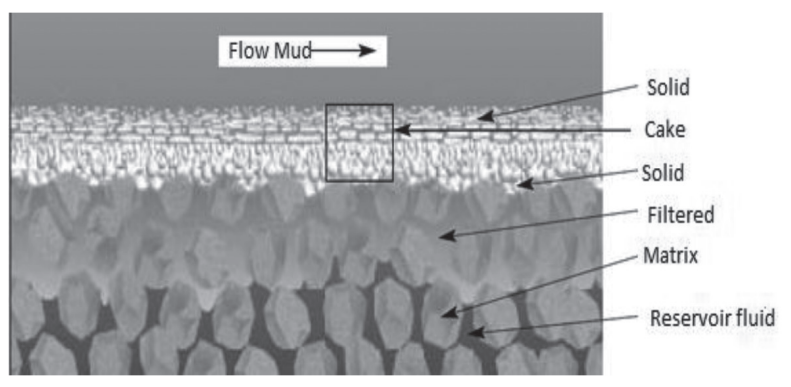

Figura 1. Características de filtración (Manual Fluidos de Perforación MI SWACO 2001)

\section{Condiciones para que se genere la filtración}

Para que pueda ocurrir la filtración se requiere de tres condiciones: Debe haber un líquido o un fluido líquido/ lechada de sólidos, debe haber un medio permeable y la presión del fluido debe ser más alta que la presión del medio permeable. Una vez se cumplen estas tres condiciones, un revoque de sólidos de lodo se acumula sobre las formaciones permeables. Mientras tanto, la fase líquida del lodo, es decir el filtrado, fluirá a través del revoque y dentro de la formación.

La filtración estática ocurre bajo condiciones estáticas, es decir en cualquier momento en que el lodo no está circulando, varios factores controlan la tasa de filtración bajo estas condiciones. La ley de Darcy puede ser usada para establecer la relación entre la tasa de filtración, la permeabilidad, superficie de la sección transversal, presión diferencial, viscosidad del filtrado y espesor del revoque (Ayala, Benítez \& Valencia, 2017). Para el flujo de filtrado a través de un revoque, la permeabilidad determinante es la del revoque, teniendo en cuenta que es mucho más baja que la permeabilidad de la formación. Por otro lado, la filtración dinámica se presenta cuando hay circulación y la presión de lodo es mayor a la presión de formación y comienza tan pronto como la broca inicia a perforar roca permeable. 
Un sobre-balance de la presión hidrostática causará el flujo inmediato del filtrado dentro de la formación a una velocidad elevada. A medida que la filtración continúa, los sólidos más grandes de lodo sellan las formaciones porosas y un revoque empieza a formarse - bajo condiciones dinámicas (Manual Fluidos de Perforación MI SWACO 2001).

\section{Modelo matemático implementado}

El modelo matemático que se utilizará para estimar las pérdidas de circulación proviene de un balance de masa en coordenadas radiales como se muestra a continuación (Escobar, 2003).

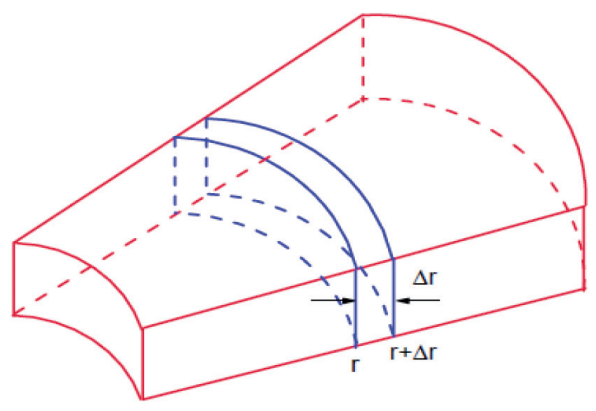

Figura 2. Elemento sobre el cual se aplica el balance de masa (Escobar, 2003)

masa que entra - masa que sale = masa que se acumula

Expresión para determinar el flujo másico que entra y que después de cierto tiempo sale de la sección analizada, se utiliza la ley de Darcy para el caudal y se multiplica por la densidad

$$
\begin{gathered}
d m=\operatorname{caudal}(Q) * \operatorname{densidad}(\rho) * d t \\
Q=2 \pi * r * h * \frac{k}{\mu} \frac{\partial p}{\partial r} \\
d m=2 \pi * r * h * \frac{k}{\mu} \frac{\partial p}{\partial r} * \rho * d t
\end{gathered}
$$

Expresión para determinar la masa que se acumula $d m=(\text { Volumen poroso } * \text { densidad })_{t+\Delta t}-$ $(\text { Volumen poroso } * \text { densidad })_{t}$

$d m=(\text { Area superficiald } r * \rho)_{t+\Delta t}-$

(Area superficial $* d r * \rho)_{t}$

$$
d m=(2 \pi * r * h * \emptyset * d r * \rho)_{t+\Delta t}-(2 \pi * r * h * \rho * \emptyset * d r)_{t}
$$

Remplazando las Ecuaciones 7 y 4 en la Ecuación 1 se obtiene la Ecuación 8 de balance de masa

$$
\begin{aligned}
& \left(2 \pi * h * r * \frac{k}{\mu} \frac{\partial p}{\partial r} * \rho * d t\right)_{r+\Delta r}-\left(2 \pi * h * r * \frac{k}{\mu} \frac{\partial p}{\partial r} * \rho * d t\right)_{r}= \\
& (2 \pi * r * h * \emptyset * d r * \rho)_{t+\Delta t}-(2 \pi * r * h * \varnothing * d r * \rho)_{t}
\end{aligned}
$$

Factorizando $d t$ y $d r$ y simplificando $2 \pi h$ se obtiene la Ecuación 9

$$
\frac{\left(\left(r * \frac{k \partial p}{\mu \partial r} * \rho\right)_{r+\Delta r}-\left(r * \frac{k \partial p}{\mu \partial r} * \rho\right)_{r}\right)}{d r}=\frac{(r * \emptyset * \rho)_{t+\Delta t}-(r * \emptyset * \rho)_{t}}{d t}
$$

Aplicando la definición de derivada a los dos terminos se obtiene la Ecuación 10

$$
\frac{1}{r} \frac{d}{d r}\left(r * \rho * \frac{k}{\mu} \frac{\partial p}{\partial r}\right)=\frac{d(\rho * \varnothing)}{d t}
$$

Derivando el término de la derecha se obtiene la Ecuación 11

$$
\frac{1}{r} \frac{d}{d r}\left(r * \rho * \frac{k}{\mu} \frac{\partial p}{\partial r}\right)=\frac{\partial \varnothing}{\partial t} * \rho+\frac{\partial \rho}{\partial t} * \varnothing
$$

Partiendo de la ecuacion de compresibilidad $c=-\frac{1}{V} \frac{\partial v}{\partial p}=\frac{1}{\rho} \frac{\partial \rho}{\partial p}$ se encuentra un término que represente el la densidad en función de la presión y dado que la porosidad es constante, el termino $\partial \emptyset / d t * \rho$ es cero

$$
\frac{\partial \rho}{\partial t}=\rho * c_{r} * \frac{\partial p}{\partial t}
$$

Remplazando la Ecuación 12 en la Ecuación11

$$
\frac{1}{r} \frac{d}{d r}\left(r * \rho * \frac{k}{\mu} \frac{\partial p}{\partial r}\right)=\varnothing * \rho * c_{r} * \frac{\partial p}{\partial t}
$$

Despejando las constantes y agrupándolas en un solo término se obtiene la Ecuación 14

$$
\frac{1}{r} \frac{d}{d r}\left(r * \rho * \frac{\partial p}{\partial r}\right)=\frac{\rho * c_{r} * \mu * \varnothing}{k} \frac{\partial p}{\partial t}
$$

Aplicando la regla de la cadena al término de la izquierda

$$
\frac{\rho}{r} \frac{d}{d r}\left(\mathrm{r} * \frac{\partial p}{\partial r}\right)+\frac{\mathrm{r}}{r} \frac{\partial p}{\partial r} \frac{d \rho}{d r}=\frac{\rho * c_{r} * \mu * \emptyset}{k} \frac{\partial p}{\partial t}
$$

Remplazando la Ecuación 12 en la 15 se obtiene

$\frac{\rho}{r} \frac{d}{d r}\left(\mathrm{r} * \frac{\partial p}{\partial r}\right)+\frac{\mathrm{r}}{r} \frac{\partial P}{\partial r} \frac{\partial p}{\partial t} * \rho * c_{r}=\frac{\rho * c_{r} * \mu * \emptyset}{k} \frac{\partial p}{\partial t}$ 
Suponiendo que las variaciones de presión son pequeñas, el término $\frac{r}{r} \frac{\partial p}{\partial r} \frac{\partial p}{\partial t} * \rho * c_{r}$ se aproxima a cero, simplificando la Ecuación 16 se obtiene

$$
\frac{1}{r} \frac{d}{d r}\left(\mathrm{r} * \frac{\partial p}{\partial r}\right)=\frac{c_{r} * \mu * \emptyset}{k} \frac{\partial p}{\partial t}
$$

Posteriormente se aplica la regla del producto al término de la izquierda y se obtiene la Ecuación 18

$$
\frac{1}{r} \frac{d p}{d r}+\frac{\partial^{2} p}{\partial r^{2}}=\frac{c_{r} * \mu * \varnothing}{k} \frac{\partial P}{\partial t}
$$

El modelo anterior sirve para modelar flujo de fluidos en medios porosos en coordenadas radiales, este modelo es similar al utilizado para estimar la presión de poro mediante el modelamiento de cuencas 1D (Vargas, Calderón, Mateus, Corzo \& Acevedo, 2014) si los parámetros están en unidades de campo se requiere de un factor de conversión como se muestra a continuación, esta constante es tomada de la literatura (Escobar, 2003).

$$
\frac{1}{r} \frac{d p}{d r}+\frac{\partial^{2} p}{\partial r^{2}}=\frac{\mathrm{c}_{r} * \mu * \varnothing}{0.0002637 k} \frac{\partial p}{\partial t}
$$

La Ecuacion 19 puede ser llevada a la solución de la línea fuente con un procedimientos matemáticos documentados en los trabajos de (Escobar, 2003) (Lee, 1982). En esos trabajos evidencian la aproximación analítica de la ecuación 19 mostrada en la Ecuación 20. Esta ecuación es utilizada para determinar cambios de presión en la cara del pozo en función de un caudal de filtrado y parámetros de la formación, los cuales están en unidades de campo.

$$
P_{w}=P_{i}-1626 * \frac{q * B * \mu}{k * h}\left(\log \frac{k * t}{\phi * \mu * c_{t} * r_{w}^{2}}+0.868 * s-3.227\right)
$$

\section{Metodología de trabajo}

La Ecuación 20 tiene estructura analítica por tal motivo no es necesario implementar métodos numéricos, para solucionarlo hay que verificar cual de sus terminos son constantes y cuales varía con la profundidad.

\section{Parámetros constantes}

Parámetros como el espesor, el daño, el radio del pozo y la tasa de pérdidas pérmitida son constantes en la seccion de estúdio al igual que la compresibilidad de la formación por tanto pueden tener un valor respresentativo para el estudio.

\section{Parámetros variables}

Algunos de los parámetros presentados en la Ecuación 20 deben ser estimados en función de la profundidad como es el caso de la porosidad, permeabilidad y viscosidad, a continuación se mostrarán algunas correlaciones utilizadas para estimar estos parámetros

\section{Porosidad}

$$
\begin{gathered}
\emptyset_{s}=\frac{\Delta t_{l o g}-\Delta t_{m a}}{\Delta t_{f l}-\Delta t_{m a}} \\
\emptyset_{s}=0,7 * \frac{\Delta t_{l o g}-\Delta t_{m a}}{\Delta t_{l o g}}
\end{gathered}
$$

Las ecuaciones de porosidad son propuestas por Wyllie (1956) y el método RHG revisadas en el trabajo realizado por Asquith \& Krygowski (2004), estas ecuaciones están en función del registro sónico y constantes estipuladas en la literatura en lo referente al tiempo de transito en fluidos y matriz de la roca. Para ilustrar el comportamiento de estas correlaciones se tomó como ejemplo un pozo Colombiano al que se le aplicará la metodología propuesta, en la Figura 3 se observa el comportamiento de las correlaciones $21 \mathrm{y}$ 22. Es de resaltar que esta propiedad puede ser estimada mediante elregistro de densidad o leida directamente del registro neutron.

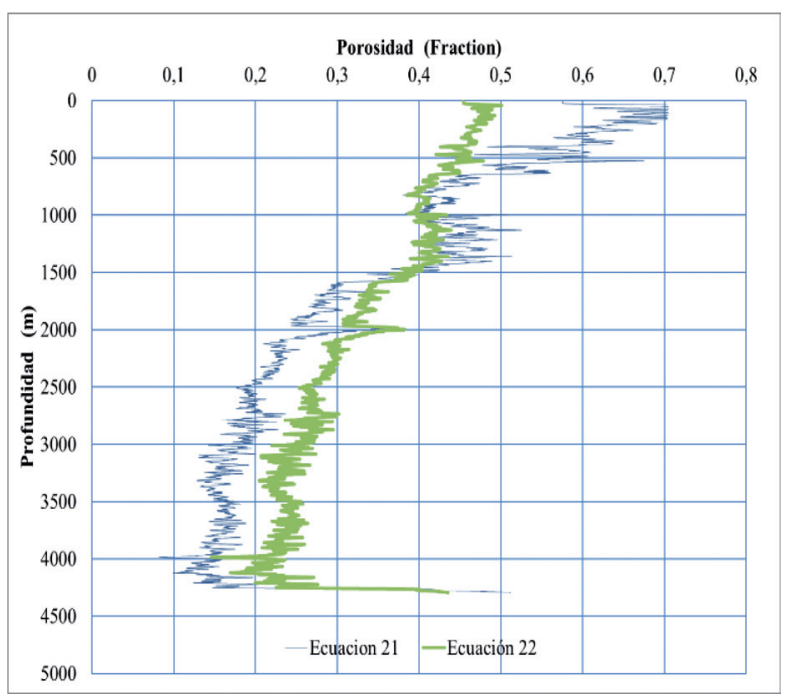

Figure 3. Perfil de porosidad en función del registro sónico usando las correlaciones 21 y 22

En la Figura 3 se observan dos perfiles de porosidad, para la solución del modelo matemático implementado para estimar perdidas de filtrado en esta investigación, se utilizó el perfil de la Ecuación 22 que parte de valores alrededor de 0.5 de porosidad lo cual se acerca más 
a la realidad ya que un empaquetamiento perfecto, la porosidad máxima que puede alcanzar una roca es $47 \%$.

\section{Permeabilidad}

La permeabilidad es una de las variables más importantes del modelo propuesto, esta variable determina si hay o no perdidas de filtrado. Se hizo una revisión de correlaciones presentadas en la literatura e implementadas en la estimación de la permeabilidad las cuales se presentan en la Tabla 1.

Tabla 1. Correlaciones utilizadas para estimar la permeabilidad

\begin{tabular}{|c|c|c|c|}
\hline AUTOR & CORRELACION & & PARÁMETROS \\
\hline $\begin{array}{l}\text { England, Mackenzie, } \\
\text { Mann \& Quigley } \\
\text { (1987) }\end{array}$ & $k=A * \varnothing^{8}$ & (23) & $\begin{array}{l}\text { Donde A es una constante que depende de la litología la } \\
\text { cual es } 4 * 10-15 \mathrm{~m}^{2} \text { para shale y } 4 * 10-12 \mathrm{~m}^{2} \text { para limolitas } \\
\text { a menos que se indique lo contrario. }\end{array}$ \\
\hline Berg (1975) & $k=0.084 * d^{2} * \varnothing^{5.1}$ & (24) & $\begin{array}{l}\text { Donde "d" es el promedio del diámetro de grano en } \\
\text { metros el cual según Walderhaug (1996) en tamaño puede } \\
\text { estar alrededor de } 2 * 10-5 \mathrm{~m} \text {. }\end{array}$ \\
\hline $\begin{array}{l}\text { Ungerer, Burrus, } \\
\text { Doligez, Chenet \& } \\
\text { Bessis (1990) }\end{array}$ & $\begin{array}{l}k=\frac{0.2 * \varnothing^{3}}{S^{2} *(1-\varnothing)^{2}} \text { si } \varnothing^{\prime}>10 \% \\
k=\frac{20 * \varnothing^{5}}{S^{2} *(1-\varnothing)^{2}} \text { si } \varnothing^{\prime}<10 \%\end{array}$ & (25) & $\begin{array}{c}\text { Donde es la porosidad efectiva para el flujo de fluidos y } \\
\mathrm{S} \text { es el área de superficie de la matriz de la roca en } \mathrm{m} 2 / \\
\mathrm{m} 3 \text { (área por unidad de volumen) el valor de } \mathrm{S} \text { depende de } \\
\text { la litología }\end{array}$ \\
\hline Dutta (1987) & $k=0.0034 *\left(\frac{\varnothing}{1-\varnothing}\right)^{5} * 10^{-15}$ & (26) & Donde la permeabilidad esta en $\mathrm{m}^{2}$ \\
\hline Luo \& Vasseur (1992) & $k=\lambda * \varnothing^{5}$ & (27) & $\begin{array}{l}\text { Para sedimentos arcillosos } \lambda \text { varía entre } 10^{-3} \text { y } 10^{-7} \text { y el } \\
\text { resultado está en Darcy }\end{array}$ \\
\hline $\begin{array}{l}\text { Lin \& Salisch, H. A. } \\
\text { (1994). }\end{array}$ & $k=0.136 * \frac{\varnothing^{4.4}}{S_{w}^{2}}$ & $(28)$ & $\begin{array}{l}\text { Donde Sw es la saturación de agua irreducible (\%) los } \\
\text { resultados están en } \mathrm{mD} \text {. }\end{array}$ \\
\hline
\end{tabular}

Continuando con las estimación de parámetros para solucionar el modelo propuesto para estimar perdidas de filtrado (Ecuación 20), se implementaron las correlaciones anteriores en función del perfil de porosidad de la Ecuación 22 para obtener la Figura 4 la cual muestra todos los perfiles de permeabilidad de la Ecuación 23 a la 28. De estos modelos se seleccionó la ecuación 27 por su amplio rango de aplicción según el contenido de arcilla, además a sido aplicada con exito en otros trabajos como Vargas et al 2014.

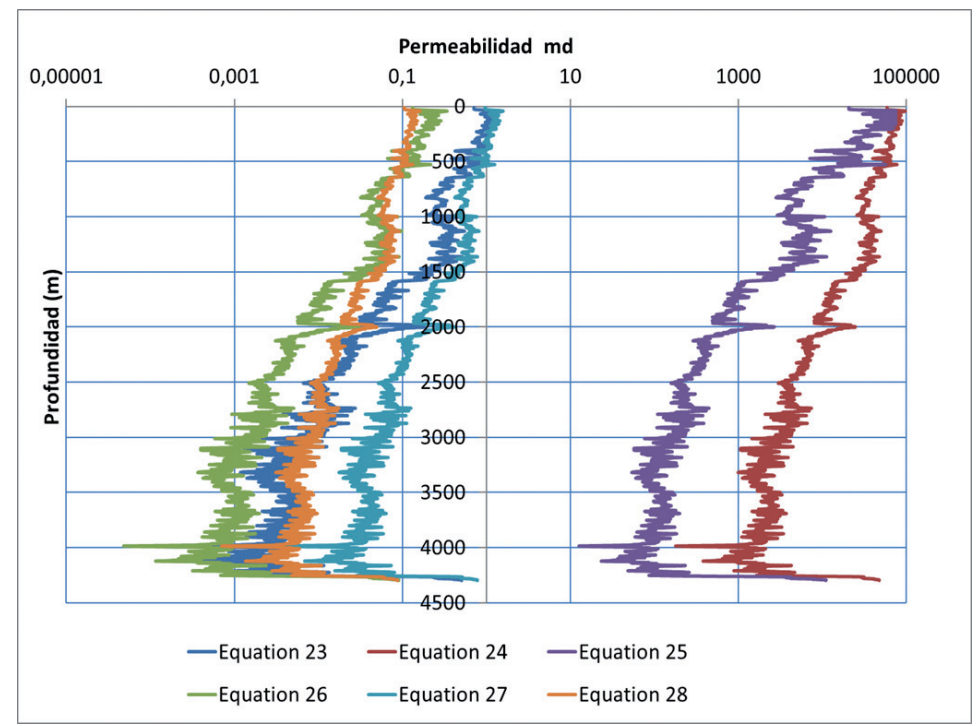

Figura 4. Perfiles de permeabilidad en función de la porosidad para las Ecuaciones de 23 a 28. 


\section{Viscosidad}

El siguiente parámetro a estimar para solucionar la Ecuación 20 es la viscosidad, ésta varía con la temperatura (Mercer, Pinder \& Donalson, 1975), para este caso el filtrado es agua y está a grandes profundidades y altas temperaturas, la viscosidad disminuye como se muestra a continuación.

$$
\begin{gathered}
\frac{1}{\mu}=\left(5.38+3.8 A-0.26 A^{3}\right) * 10^{3} \\
A=\frac{\mathrm{T}-150^{\circ} \mathrm{C}}{100^{\circ} \mathrm{C}}
\end{gathered}
$$

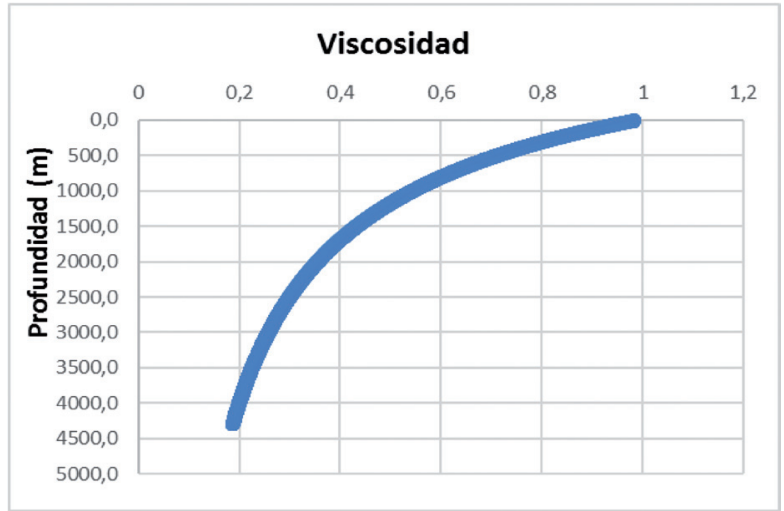

Figura 5. Perfiles de viscosidad

Luego de tener todos los parámetros, los constantes y los que varían en profundidad se soluciona de forma analítica la Ecuación 20. En las tablas 2 y 3 se tiene la información complementaria para generar la figura 6 .

Tabla 2. Propiedades del lodo (ICP)

\begin{tabular}{lllr}
\hline \multicolumn{4}{c}{ Propiedades del lodo } \\
\hline Parámetro & & Unidad & \multicolumn{1}{c}{ Valor } \\
\hline Bo & Factor volumétrico & $\mathrm{bb} / \mathrm{STB}$ & 1 \\
\hline$\mu \mathrm{o}$ & Viscosidad & $\mathrm{cp}$ & 1 \\
$\mathrm{t}$ & Tiempo & $\mathrm{hr}$ & 500 \\
Q & Caudal & $\mathrm{gpm}$ & 4,00 \\
Q & Caudal & $\mathrm{Bb} / \mathrm{D}$ & 137,142857 \\
Q & Caudal & $\mathrm{Bb} / \mathrm{h}$ & 5,71428571 \\
Q cada 0.5 pie & Caudal & $\mathrm{Bb} / \mathrm{D}$ & 0,00489796 \\
Pwf & Presión de forma & $\mathrm{psi}$ & \\
Cf & Compresibilidad & $1 / \mathrm{psi}$ & $7,25 \mathrm{E}-05$ \\
\hline
\end{tabular}

Tabla 3. Propiedades de la formación (ICP)

\begin{tabular}{lllr}
\hline \multicolumn{4}{c}{ Parámetros de la formación } \\
\hline Parámetro & & \multicolumn{1}{c}{ Unidad } & Valor \\
\hline $\mathrm{Pi}$ & Presión inicial & $\mathrm{psi}$ & \\
$\mathrm{k}$ & Permeabilidad & $\mathrm{md}$ & \\
$\mathrm{h}$ & Espesor & $\mathrm{ft}$ & 0,009144 \\
Фo & Porosidad & Adimensional \\
gra T & Gradiente & ${ }^{\circ} \mathrm{C} / \mathrm{ft}$ & 0,2 \\
Swr & Saturación & Adimensional & 2 \\
s & Daño & Adimensional & 6 \\
rw & Radio del pozo & in & 0,5 \\
\hline rw & Radio del pozo & ft & \\
\hline
\end{tabular}

\section{Resultados}

Luego de aplicar la Ecuación 20 y solucionarla utilizando los perfiles de porosidad, permeabilidad, viscosidad y datos de las Tablas 2 y 3 se obtiene el siguiente perfil que muestra la presión máxima permitida con pérdidas de filtrado controladas, cabe resaltar que esta tasa de filtrado hace referencia a un una tasa máxima de pérdidas que no afecte los costos y no genere inestabilidad de pozo. La presión permitida se ve en la Figura 6 .

En la Figura 6 se observa la curva de presión de poro (datos suministrados) y de presión permitida (Ecuación 20) manteniendo unas pérdidas de filtrado controladas, mientras el peso del lodo se mantenga en ese rango se lograran evitar pérdidas de filtrado excesivas e influjos de la formación.

El modelo implementado en esta investigación para perdidas de filtrado controladas depende de características de la formación y de parámetros del lodo, algunas son más importantes que otras por lo tanto su cálculo debe ser más cuidadoso, para determinar cuál variable influye más se realizó un análisis de sensibilidad como se observa en la Figura 7.

Los resultados del análisis de sensibilidad muestran que la permeabilidad es la variable de mayor importancia en el modelo, esto se debe a que esta variable determina la facilidad que tiene la roca para dejar pasar un fluido, la segunda variable más importante es la viscosidad la cual está relacionada con la facilidad que tiene el fluido para moverse. El caudal también presenta gran importancia pero para el modelo propuesto será constante ya que se basa en pérdidas controladas; en orden de importancia las demás variables son: daño a la formación, tiempo, radio del pozo y finalmente la compresibilidad. 
Modelo matematico para determinar presión permitida de pérdidas de filtrado de lodo controladas

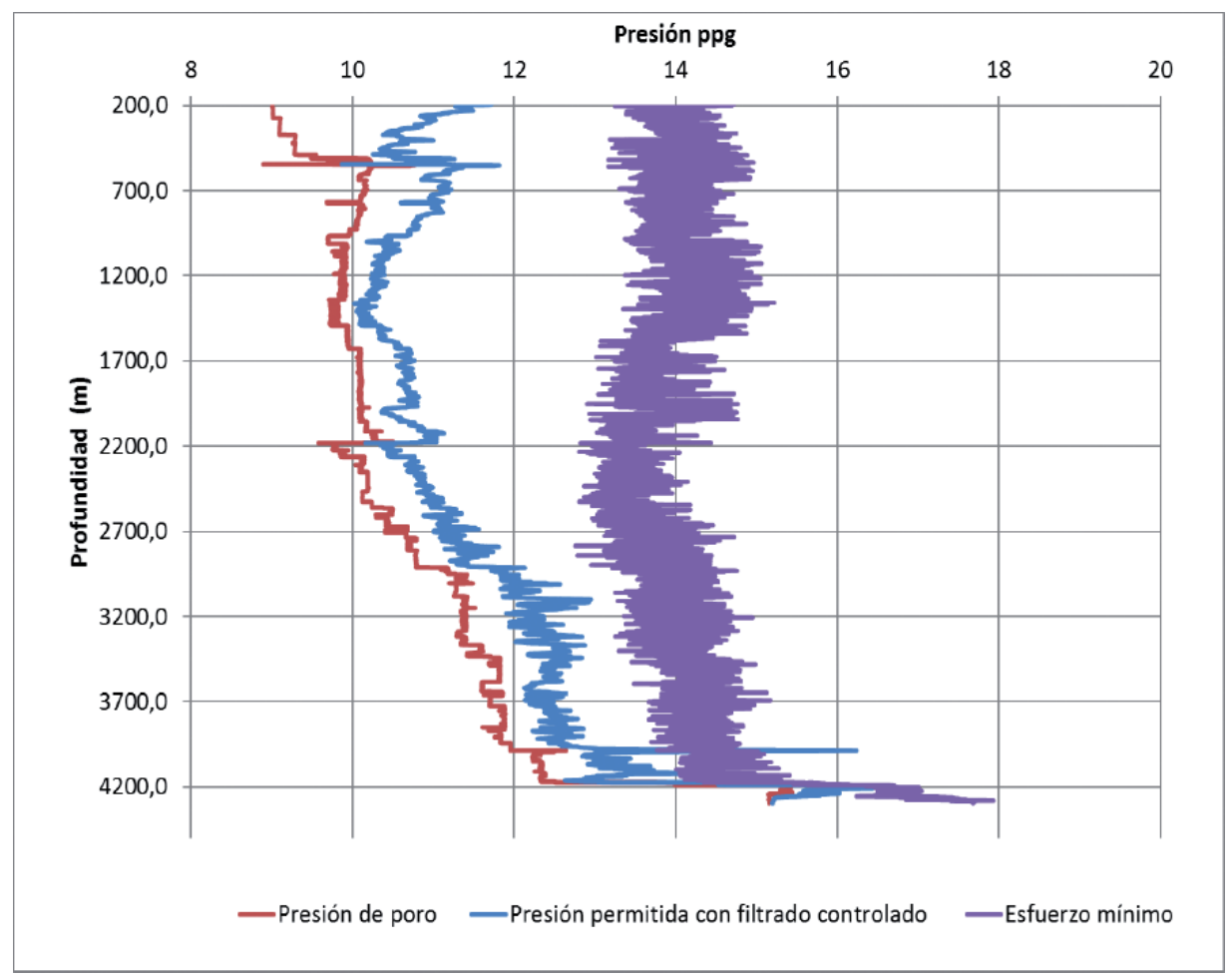

Figura 6. Perfil de presión con pérdidas de filtrado controladas

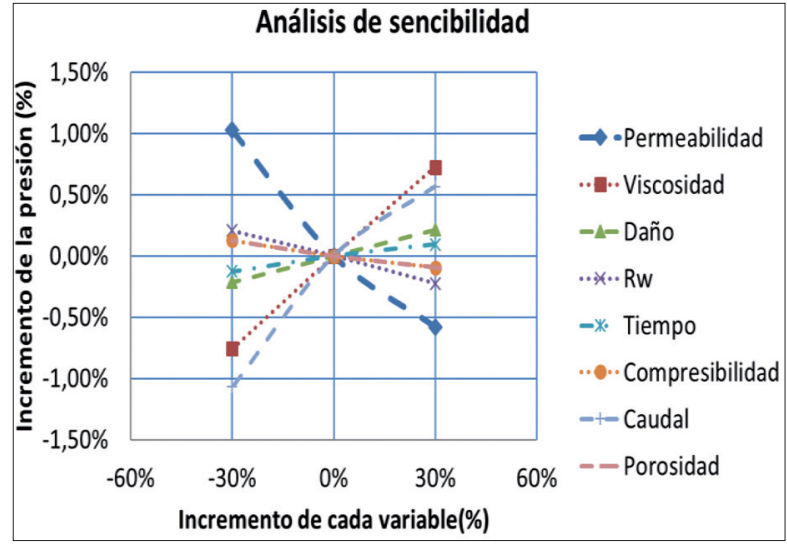

Figura 7. Análisis de sensibilidad.

\section{Conclusiones}

El modelo usado en análisis de presión se puede adaptar e implementar para estimar la presión permitida del lodo durante la perforación y controlar el filtrado de este fluido hacia la formación y de esta forma, adicionar una nueva curva a la ventana de estabilidad

El modelo matemático implementado dio como resultado un perfil de presión para pérdidas controladas de filtrado entre los rangos permitidos que son el esfuerzo mínimo y la presión de poro, por lo tanto se puede afirmar que este modelo propuesto no solo sirve para análisis de pruebas de presión de la formación a la cara del pozo si no que se puede ajustar para perdidas de filtrado desde el pozo hacia la formación.

El perfil de presión generado para pérdidas controladas, da una idea del peso del lodo que se puede utilizar para perforar el cual estará en el rango de aplicación de estabilidad y ayudará a disminuir la pérdidas de filtrado.

Luego del análisis de sensibilidad, la variable que más afecta la presión máxima para pérdidas de filtrado controlada es la permeabilidad, según las correlaciones analizadas, estas dependen de porosidad, por lo tanto si no se tienen datos de laboratorio, es conveniente hacer los cálculos de porosidad lo más acertado posible para evitar errores en las curvas resultantes.

La segunda variable que más afecta es la viscosidad, por lo tanto, se debe hacer un análisis del filtrado de lodo ya que las trazas de bentonita $u$ otros aditivos puede aumentar la viscosidad del agua, cabe resaltar que la viscosidad varía en función de la temperatura a medida que aumente la profundidad mediante un modelo matemático. 


\section{Nomenclature}

$\begin{array}{lll}\mathrm{Pw} & \text { Presión de fondo } & \mathrm{Mp} \\ \mathrm{Pi} & \text { Presión de poro } & \mathrm{Mp} \\ \mathrm{B} & \text { Factor volumétrico } & - \\ \mathrm{k} & \text { Permeabilidad } & \mathrm{md} \\ \mathrm{h} & \text { Espesor } & \mathrm{m} \\ \varnothing & \text { Porosidad } & - \\ \text { gra T } & \text { Temperatura } & { }^{\circ} \mathrm{C} / \mathrm{km} \\ \mathrm{Swr} & \text { Saturación } & - \\ \mathrm{s} & \text { Daño } & - \\ \mathrm{rw} & \text { Radio del pozo } & \mathrm{m} \\ \mathrm{Bo} & \text { Factor volumétrico } & \mathrm{bb} / \mathrm{STB} \\ \mu \mathrm{o} & \text { Viscosidad } & \mathrm{cp} \\ \mathrm{t} & \text { Tiempo } & \mathrm{hr} \\ \mathrm{Q} & \text { Caudal } & \mathrm{Bb} / \mathrm{D} \\ \mathrm{Cf} & \text { Compresibilidad } & 1 / \mathrm{Mp} \\ \Delta t l o g & \text { Tiempo del registro } & \mathrm{us} / \mathrm{ft} \\ \Delta t_{f l} & \text { Tiempo a través del fluido } & \mathrm{us} / \mathrm{ft} \\ \Delta t_{m a} & \text { Tiempo a través de la matriz } & \mathrm{us} / \mathrm{ft}\end{array}$

\section{Referencias}

1. Asquith, G. \& Krygowski, D. (2004) Basic well log analysis: $A A P G$ Methodos in exploration 16, p 40.

2. Ayala, D., Benítez, A., \& Valencia, R. (2017). Optimización de la Tasa de Penetración mediante el análisis de las vibraciones al perforar, caso de estudio Ecuador. Revista Fuentes, 15(1), 27-40.

3. Ayala, F. E. B., Gómez, J. Q., \& León, E. A. (2011). Estudio de factibilidad del uso del biodiesel como fase contínua en lodos de perforación de emulsión inversa. Fuentes: El reventón energético, 9(1), 6 .

4. Ayala, D., Torres, H., Valencia, R., \& Loaiza, M. (2016). Impacto del Tiempo no Productivo en operaciones de perforación y análisis de los datos mediante la prueba de Chicuadrado. Revista Fuentes, 14(2), 5-18.

5. Berg, R. R. (1975). Capillary pressures in stratigraphic traps. American Association of Petroleum Geologists'Bulletin, 59, 939-56.

6. Campana, D. E. A., \& Tapia, R. A. V. (2017). Evaluación cualitativa de la limpieza de hoyo en pozos de alta inclinación-alto desplazamiento en la Cuenca Oriente. Fuentes: El reventón energético, 15(2), 49-56.

7. Dutta, N.C. (1987). Fluid flow in low permeable porous media. In: Doligez, E. (ed.) Migration of Hydrocarbons in Sedimentary Basins, 567595. Editions Technip, Paris.

8. Escobar. F. (2003) Análisis moderno de presiones. Neiva, Huila.

9. England, W.A., Mackenzie, A.S., Mann, D.M. \& Quigley, T.M. (1987): The movement and entrapment of petroleum fluids in the subsurface. Journal of the Geological Society, London 144. 327-347.

10. Ghalambor, A., Salehi, S., Shahri, M. P. \& Karimi, M. (2014). Integrated Workflow for Lost Circulation Prediction. Society of Petroleum Engineers. doi:10.2118/168123-MS.

11. John Lee. "Well Testing". SPE textbook series Vol. 1. 1982.

12. Lin, J. L. \& Salisch, H. A. (1994). Determination From Well Logs of Porosity and Permeability in a Heterogeneous Reservoir. Society of Petroleum Engineers. doi:10.2118/28792-MS.

13. Luo, X. \& Vasseur, G. (1992) Contributions of compaction and aquathermal pressuring to geopressure and the influence of environmental conditions. American Association of Petroleum Geologists Bulletin 76, 1550-1559.

14. Mercer, J. W., G. F. Pinder, \& I. G. Donalson, (1975), A Galerkinfinite element analysis of the hydrothermal system at Wairakei, New Zealand: Journal of Geophysical Research, v. 80, p. 2608-2621.

15. Ungerer, P., Burrus, J., Doligez, B., Chenet, P. \& Bessis, F. (1990) Basin evaluation by integrated two-dimensional modeling of heat transfer, fluid flow, hydrocarbon gerneration and migration. AAPG Bulletin, 74,309-335.

16. Parn-anurak, S. \& Engler, T. (2005) Modeling of fluid filtration and near-wellbore damage along a horizontal well. Journal of Petroleum Science and Engineering, Vol 46 (3), Pages 149-160, 
ISSN 0920-4105, http://dx.doi.org/10.1016/j. petrol.2004.12.003.

17. Portilla, H. E., Suárez, D. F., \& Corzo, R. (2012). Metodología para la optimización de parámetros de perforación a partir de propiedades geomecánicas. Revista Fuentes, 10(2).

18. Sanfillippo, F., Brignoli, M., Santarelli, F. J. \& Bezzola, C. (1997). Characterization of conductive fractures while drilling. Paper SPE 38177 presented at the SPE European Formation Damage Conference, 2-3 June, The Hague, Netherlands.
19. Shahri, M. P., Zeyghami, M. \& Majidi, R. (2012) Investigation of fracture ballooning-breathing using an exponential deformation law and Herschel-Bulkley fluid model. Special Topics \& Reviews in Porous Media-An International Journal, 3(4), 341-351.

20. Vargas, D. A., Calderón, Z. H., Mateus, D. C., Corzo, R.. \& Acevedo, O. J. (2014). Mathematical Model to Quantify the Contribution of Thermal Stresses in Pore Pressure, Additional to the Compaction Effect. International Society for Rock Mechanics.

Recepción: 5 de febrero de 2018

Aceptación: 27 de abril de 2018 